\title{
Propagators, Running Coupling and Condensates in Lattice QCD
}

\author{
Attilio Cucchieri and Tereza Mendes \\ Instituto de Física de São Carlos, Universidade de São Paulo, \\ C.P. 369, 13560-970, São Carlos, SP, Brazil
}

\section{Received on 9 July, 2006}

\begin{abstract}
We present a review of our numerical studies of the running coupling constant, gluon and ghost propagators, ghost-gluon vertex and ghost condensate for the case of pure $S U(2)$ lattice gauge theory in the minimal Landau gauge. Emphasis is given to the infrared regime, in order to investigate the confinement mechanisms of QCD. We compare our results to other theoretical and phenomenological studies.
\end{abstract}

Keywords: Lattice gauge theory; Monte Carlo simulation; Confinement; Running coupling constant

\section{INTRODUCTION}

The strong force - one of the four fundamental interactions of nature along with gravity, electromagnetism and the weak force - is the force that holds together protons and neutrons in the nucleus. The strong interaction is described by Quantum Chromodynamics (QCD) [1]. This description is based on a model of elementary particles — the quarks — possessing "color charge" and interacting through the exchange of gauge fields - the gluons (equivalent to the photons in the electromagnetic interaction). QCD is a quantum field theory, with local $S U(3)$ gauge symmetry, corresponding to three possible colors. The fact that the gauge group of QCD is non-Abelian implies that the gluons possess color charge and therefore interact with each other, as opposed to the photons. The only parameters of the theory are the masses of the various types (called "flavors") of quarks considered and the value of the strong coupling constant.

A unique feature of the strong force is that the particles that feel it directly — quarks and gluons - are completely hidden from us, i.e. they are never observed as free particles. This phenomenon, known as confinement, makes QCD much harder to handle theoretically than the theories describing the weak and electromagnetic forces. Indeed, the coupling constant $\alpha_{s}$ of the strong interaction becomes negligible only in the limit of small distances, or equivalently in the limit of high energy or momentum. This property is called asymptotic freedom. At larger distances (i.e. smaller energies) there is an increase in the intensity of the interaction and it is believed that the force of attraction between quarks is constant, for sufficiently large distances, determining the confinement of quarks and gluons inside the hadrons. The fact that $\alpha_{s}$ is not negligible at low energies makes the study of important phenomena such as the mechanism of quark and gluon confinement, the hadron mass spectrum and the deconfining transition at finite temperature inaccessible to calculations using perturbation theory. These phenomena must therefore be studied in a nonperturbative way.

The nonperturbative study of QCD is possible in the lattice formulation of the theory, introduced by Wilson in 1974 [2]. This formulation offers a convenient nonperturbative regularization, preserving the theory's gauge invariance. The essential ingredients for the lattice formulation are: 1) path-integral quantization, 2) continuation to imaginary or Euclidean time and 3) lattice regularization (given by the discretization of space-time). The combination of the first two ingredients makes the theory equivalent to a model in classical statistical mechanics: indeed, in Euclidean space a path integral for the quantum theory is equivalent to a thermodynamic average for the corresponding statistical mechanical system. For QCD, the square of the bare coupling constant $g_{0}$ of the field theory corresponds directly to the temperature $1 / \beta$ of the statistical mechanical model.

The third ingredient — the lattice discretization - represents an ultraviolet regularization. In fact, the lattice spacing $a$ corresponds to a high-momentum cutoff, since momenta higher than $\sim 1 / a$ cannot be represented on the lattice. In this way the ultraviolet divergences, appearing in the calculation of physical quantities, are suppressed and the theory is well defined. Of course, in order to recover the continuum-space theory we must take the limit $a \rightarrow 0$. In this process it is necessary to "tune" the bare parameters of the theory in such a way that physical quantities converge to finite values, which can then be compared to experiment. In particular, in the limit $a \rightarrow 0$, a correlation length $\xi$ measured in units of the lattice spacing, i.e. $\xi / a$, must go to infinity. In other words, the lattice theory considered must approach a critical point, i.e. a second-order phase transition. Thus, the study of the continuum limit in quantum field theories on the lattice is analogous to the study of critical phenomena in statistical mechanics. The correspondence between Euclidean field theories and classical statistical mechanics allows the application of usual statistical-physics methods to the study of QCD. In particular, one may perform numerical simulations by Monte Carlo methods, which are based on a stochastic description of the systems considered [3].

Despite the similarity of the methods, the Monte Carlo simulation of gauge theories is much more complex than in the case of the usual statistical mechanical models, requiring great computational effort and specific numerical techniques for the production of the data. Moreover, we must consider three limits in order to obtain the desired physical results from the simulation data: 1) the infinite-volume limit (or thermodynamic limit), 2) the continuum limit (i.e. the value of $a$ must be sufficiently small when compared to the relevant distance for the problem) and 3) the chiral limit (in order to consider physical values for the masses of the light quarks). The above limits are not independent, since to get to the continuum limit and 
to be able to consider small masses for the quarks one needs a sufficiently large number of lattice points (corresponding to a small enough lattice spacing and to a large enough physical size of the lattice), which increases considerably the computational effort.

The study of lattice QCD constitutes a so-called Grand Challenge computational problem [4]. Indeed, simulations of full QCD - i.e. including effects of dynamical fermions - for quark masses in the region of physical values are still extremely slow and they are in general carried out on supercomputers, involving the effort of large collaborations such as the UKQCD in the United Kingdom and the JLQCD in Japan. Also, several research groups have built QCD-dedicated computers, using parallel architecture. Examples are the Hitachi/CP-PACS machine at the University of Tsukuba in Japan [5], the QCDSP and QCDOC machines at Columbia University in the USA [6], and the APE machines [7] at various research centers in Europe. These computers range from about 1 to 10 teraFLOPS. In addition to these large projects, many groups base their simulations on clusters of workstations or personal computers (PC's) [8]. These systems do not yet provide the same efficiency in parallelization as the machines with parallel architecture, but their cost is much lower. In addition to the computational power, the numerical and analytical techniques used in the simulations and in the interpretation of the produced data are of great importance in the field. Significant progress has been achieved through the development of more efficient simulation algorithms, new methods for interpolation and extrapolation of the numerical data and a better understanding of the systematic effects to which the simulation may be subject, such as finite-volume effects and discretization errors. Progresses in the field are reported annually at the Lattice conference [9].

Despite the great computational difficulty, numerical studies of QCD have already provided important contributions to the study of the strong force [10]. In particular, numerical simulations of QCD are now able to produce calculations of the strong coupling constant $\alpha_{s}\left(\mu_{0}\right)$, taken at a fixed reference scale $\mu_{0}$, with precision comparable to the experimental one or better [11]. These results are presently included in the world average for this quantity [12]. Also, the mass spectrum of the light hadrons (including the two light quarks and the strange quark) has been determined (with great precision) [13] for the quenched case, in which the configurations are produced without considering effects of dynamical quarks. One does not obtain complete agreement with the experimental spectrum, but the observed discrepancies are of at most $10 \%$. Similar calculations are now being performed for the full-QCD case. Finally, lattice simulations constitute the only known evidence for the quark-deconfining transition at finite temperature [14] and its predictions are of direct interest for the current experiments in search of new states of matter in the laboratories Brookhaven and CERN.

There is presently great interest in the results of the simulations described above and one hopes to be able to solve many theoretical questions about QCD and the standard model [15]. Indeed, lattice-QCD simulations are now able to provide quantitative predictions with errors of a few percent.
This means that these simulations will soon become the main source of theoretical results for comparison with experiments in high-energy physics [16], enabling a much more complete understanding of the physics of the strong force.

\section{A. Lattice QCD at the IFSC-USP}

Since the beginning of 2001 we have been carrying out a project on numerical simulations of lattice gauge theories at the Physics Department of the University of São Paulo in São Carlos (IFSC-USP), funded by FAPESP [17]. The project included the installation of 2 PC clusters (with a total of 28 processing nodes). The resulting computer power is of approximately 40 gigaFLOPS for peak performance. We have performed production runs since July of 2001 and have started intensive parallel simulations in November 2002. Our main research topic (see Sections II-VII below) is the investigation of the infrared behavior of various propagators and vertices in Landau gauge with the goal of verifying the so-called GribovZwanziger confinement scenario $[18,19]$. In order to reduce the computational cost of the simulations, we consider the pure $S U(2)$ gauge theory, including studies in three (instead of the usual four) space-time dimensions.

Besides the topics described below, we also carry out numerical studies of: gauge-fixing algorithms [20], Gribov-copy effects [21], the chiral phase transition of QCD with two dynamical fermions [22], the equation of state of spin models with Goldstone modes [23], cluster percolation [24] and shorttime dynamics for spin models [25].

\section{CONFINEMENT SCENARIOS IN LANDAU GAUGE}

As said above, the study of the infra-red (IR) limit of QCD is of central importance for understanding the mechanism of confinement and the dynamics of partons at low energy. Despite being non-gauge-invariant, gluon and ghost propagators are powerful tools in the (non-perturbative) investigation of this limit $[26,27]$. In fact, according to the Gribov-Zwanziger $[18,28,29]$ and to the Kugo-Ojima [30] confinement scenarios in Landau gauge, the ghost propagator must show a divergent behavior in the IR limit - stronger than $p^{-2}$ - for vanishing momentum $p$. This strong IR divergence corresponds to a long-range interaction in real space, which may be related to quark confinement. At the same time, according to the former scenario, the gluon propagator must be suppressed and may go to zero in the IR limit [18, 28, 29, 31]. This would imply that the real-space gluon propagator is positive and negative in equal measure, i.e. reflection positivity is maximally violated [32, 33]. As a consequence, the Euclidean 2-point function cannot represent the correlations of a physical particle. This result may be viewed as an indication of gluon confinement [26].

These theoretical predictions have been confirmed by studies using Dyson-Schwinger equations (DSE's) [26, 27]. In particular, studies of DSE's in Landau gauge have found [3436 ] an IR behavior of the form $G(p) \sim p^{-2 \kappa-2}=p^{-2 a_{G}-2}$ for 
the ghost propagator and of the form $D(p) \sim p^{4 \kappa-2}=p^{2 a_{D}-2}$ for the gluon propagator with the same exponent $\kappa$ (i.e. with $\left.a_{G}=a_{D} / 2\right)$. In $4 \mathrm{~d}$ one usually finds $\kappa \gtrsim 0.5$ for pure $S U\left(N_{c}\right)$ gauge theory. Note that $\kappa>0.5$ implies $D(0)=0$. For the $3 d$ case the exponents are $a_{G} \approx 0.4$ and $a_{D} \approx 1.3$. Note that in the $d$ dimensional case $[34,35]$ the relation between $a_{D}$ and $a_{G}$ is given by $a_{D}=2 a_{G}+(4-d) / 2$, implying for the quantity $\alpha_{s}(p)=\left(g^{2} / 4 \pi\right) D(p) G^{2}(p) p^{6}$ the IR behavior $p^{2\left(a_{D}-2 a_{G}\right)}=p^{4-d}$. Thus, in the 4-dimensional case the running coupling $\alpha_{s}(p)$ displays and IR fixed point.

Numerical studies of lattice gauge theories confirm the IR divergence of the Landau ghost propagator [21, 37, 38] and an IR suppression of the gluon propagator. More precisely, a decreasing gluon propagator at small momenta has been obtained for the $3 d S U(2)$ Landau case using very large lattices [39] and - recently — in the $4 d S U$ (3) Landau case with the use of asymmetric lattices [40]. Similar results has also been obtained for the the equal-time three-dimensional transverse gluon propagator in $4 d S U(2)$ Coulomb gauge [41]. In this last case, one also obtains an excellent fit of the transverse propagator by a Gribov-like formula. Finally, direct support to the Gribov-Zwanziger and to the Kugo-Ojima scenarios has been presented in [42] and in [43], respectively.

Thus, the two nonperturbative approaches above seem to support the Gribov-Zwanziger and the Kugo-Ojima confinement scenarios in Landau gauge. However, the agreement between the two methods is still at the qualitative level. Moreover, recent lattice studies $[44,45]$ seem to indicate a null IR limit for $\alpha_{s}(p)$, instead of a finite nonzero value. At the same time, a study based on DSE's [46] showed that torus and continuum solutions are qualitatively different. This suggests a nontrivial relation between studies on compact and on noncompact manifolds and could have important implications for lattice studies. Also, the nonrenormalizability of the ghostgluon vertex - proven at the perturbative level [47], confirmed on the lattice [45, 48] (for $p \gtrsim 200 \mathrm{MeV}$ ) and used in DSE studies to simplify the coupled set of equations has been recently criticized in Ref. [44]. Thus, clear quantitative understanding of the two confinement scenarios is still an open problem.

\section{INFINITE-VOLUME LIMIT}

The study of the IR behavior of propagators and vertices, i.e. for momenta smaller than $1 \mathrm{GeV}$, requires careful consideration of the infinite-volume limit. Indeed, since the smallest non-zero momentum that can be considered on a lattice is given by $p_{\min } \approx 2 \pi / L-$ where $L$ is the size of the lattice in physical units - it is clear that one needs to simulate at very large lattice sizes in order to probe the small-momentum limit. The consideration of very large lattice sizes requires parallelization and high efficiency of the code in order to obtain good statistics in the Monte Carlo simulation. Thus, an optimized parallel code is of great importance [49]. Our numerical code is parallelized using MPI; for the random number generator we use a double-precision implementation of RANLUX (version 2.1) with luxury level set to 2 .

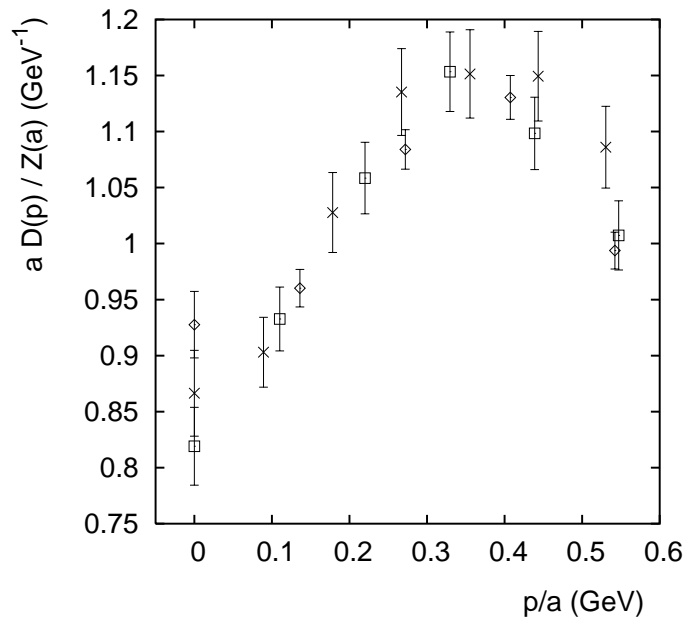

FIG. 1: Plot of the rescaled gluon propagator $a D(0) / Z(a)$ as a function of the momentum for $V=80^{3}$ and $\beta=4.2(\times), 5.0(\square), 6.0(\diamond)$. Error bars are obtained from propagation of errors.

\section{A. Very large lattice side}

In Ref. [39] we have evaluated the lattice gluon propagator $D(k)$ and study it as a function of the momentum $p(k)$ in the $3 \mathrm{~d} S U(2)$ Landau case, using data from the largest lattice side to date, i.e. up to $140^{3}$. This allowed us to consider momenta as small as $51 \mathrm{MeV}$ (in the deep IR region) and physical lattice sides almost as large as $25 \mathrm{fm}$.

In order to compare lattice data at different $\beta$ 's, we apply the matching technique described in [50], i.e. the propagators are multiplied by a factor $Z(a)$ depending on $\beta$ or, equivalently, on the lattice spacing $a$. The method works very well (see Fig. 1); indeed, data obtained using different $\beta$ values nicely collapse into a single curve. We find that the gluon propagator decreases in the IR limit for momenta smaller than $p_{\max }$, which corresponds to the mass scale $M$ in a Gribov-like propagator $D(p)=p^{2} /\left(p^{4}+M^{4}\right)$. From the plot we can estimate $p_{\text {max }}=M=0.8_{-0.1}^{+0.2} \sqrt{\sigma}=350_{-50}^{+100} \mathrm{MeV}$, in agreement with Ref. [51]. (Here $\sigma$ is the string tension.)

In Fig. 2 we plot the rescaled gluon propagator at zero momentum, namely $a D(0) / Z(a)$, as a function of the inverse lattice side $L^{-1}=1 /(a N)$ in physical units $\left(\mathrm{fm}^{-1}\right)$. We see that $a D(0) / Z(a)$ decreases monotonically as $L$ increases, in agreement with Ref. [52]. It is interesting to notice that these data can be well fitted using the simple Ansatz $d+b / L^{c}$ both with $d=0$ and $d \neq 0$ (see Figure 2). In order to decide for one or the other result one should go to significantly larger lattice sizes.

Also, in Ref. [39] we have shown that the data for the gluon propagator are well fitted by Gribov-like formulae, yielding an IR critical exponent $\kappa \approx 0.65$ in agreement with recent analytic results (see Section II). Recently [53] we have extended this analysis to the ghost propagator, considering lattice volumes up to $80^{3}$ for the coupling $\beta=4.2$. A fit to the data using the fitting function $b / p^{a}$ (in the interval $p \leq 0.5 \mathrm{GeV}$ ) gives $a=2.40(2)$. This result would imply $a_{G} \approx 0.4$, also in 
agreement with the results reported in Section II above.

\section{B. Asymmetric lattices}

Recently, very asymmetric lattices $[40,54]$ have been considered in order to explore the IR limit od QCD. As a test of this method, we have extended [55] the gluon propagator study presented in [39] [for the $3 d S U(2)$ case in minimal Landau gauge], by including results for the ghost propagators from very large lattices. At the same time, we evaluated the propagators using also asymmetric lattices, in order to verify possible systematic effects related to the use of asymmetric lattices (as suggested in [45]), by comparing the results to the ones obtained for symmetric lattices.

We find, for both propagators, clear evidences of systematic effects at relatively small momenta, i.e. $p \lesssim 1.5 \sqrt{\sigma} \approx$ $650 \mathrm{MeV}$. In particular, the gluon (respectively, ghost) propagator is less suppressed (respec. enhanced) in the IR limit when considering asymmetric lattices than for the case of symmetric lattices (see Fig. 3). This implies that the estimates for the IR critical exponents $a_{G}$ and $a_{D}$ are systematically smaller in the asymmetric case compared to the symmetric one.

Also, for the gluon propagator and considering the asymmetric lattices, one would estimate a value $M \lesssim 0.25 / a \approx 0.22$ $\mathrm{GeV}$ as a turnover point in the IR, i.e. the momentum $p_{\max }=$ $M$ for which the propagator reaches its peak. On the other hand, considering the largest (symmetric) lattice volumes, i.e. $V=140^{3}$ (see the top plot in Fig. 3), the gluon propagator is clearly a decreasing function of $p$ for $p \lesssim 0.5 / a$, corresponding to $M \lesssim 0.435 \mathrm{GeV}$. This is in agreement with the result reported in Section III A above. We thus see a difference of almost a factor 2 between the momentum-turnover point in the

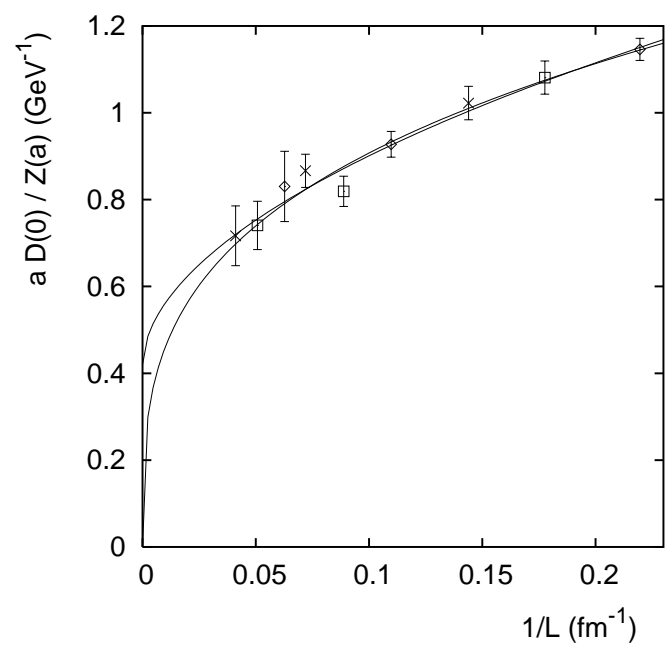

FIG. 2: Plot of the rescaled gluon propagator at zero momentum as a function of the inverse lattice side. We also show the fit of the data using the Ansatz $d+b / L^{c}$, both with $d=0$ and with $d \neq 0$. Error bars are obtained from propagation of errors.
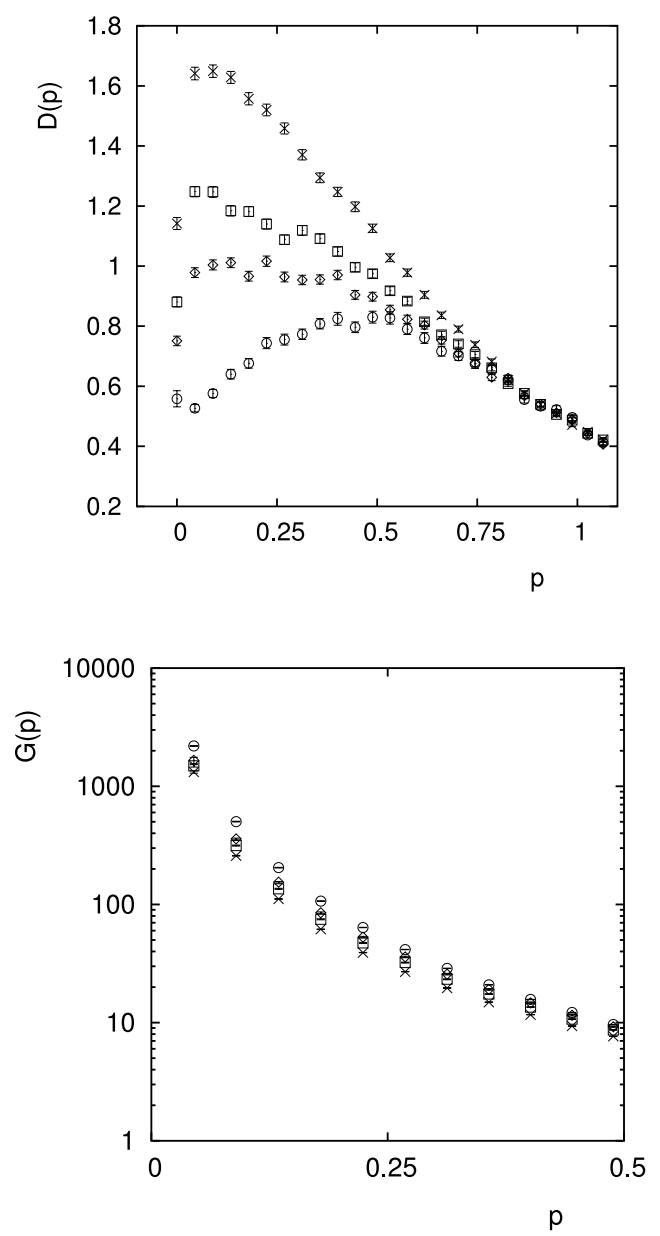

FIG. 3: Plot of the gluon propagator $D(p)$ (top) and of the ghost propagator $G(p)$ (bottom) as a function of $p$ for lattice volumes $V=$ $8^{2} \times 140(\times), 12^{2} \times 140(\square), 16^{2} \times 140(\diamond)$ and $V=140^{3}(\bigcirc)$. All quantities are in lattice units. Note the logarithmic scale on the $y$ axis in the bottom plot. Errors represent one standard deviation.

symmetric and asymmetric cases.

Finally, we have seen that the extrapolation to infinite volume of results obtained using asymmetric lattices is also most likely affected by systematic errors. We conclude that, even though using an asymmetric lattice does not modify the qualitative behavior of the two propagators, one should be careful in extracting quantitative information from such studies.

\section{REFLECTION-POSITIVITY VIOLATION}

The relation between reflection positivity and Euclidean correlation functions can be made explicit by considering the spectral representation $[26,56]$

$$
D(p)=\int_{0}^{\infty} d m^{2} \frac{\rho\left(m^{2}\right)}{p^{2}+m^{2}}
$$




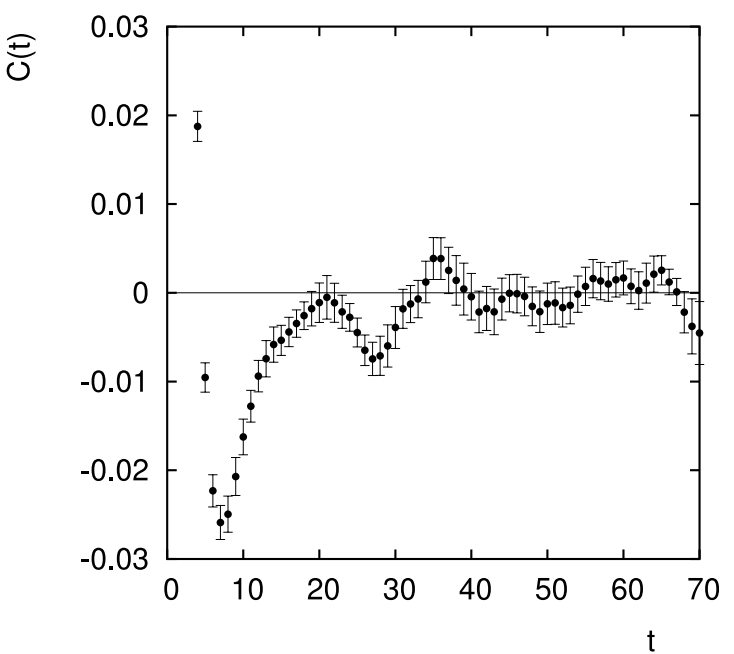

FIG. 4: Real-space propagator $C(t)$ as a function of $t$ for coupling $\beta=5.0$ and lattice volume $V=140^{3}$. Errors have been evaluated using bootstrap with 1000 samples. All quantities are in lattice units.

for the Euclidean propagator in momentum space. Then, the statement of reflection positivity is equivalent to a positive spectral density $\rho\left(\mathrm{m}^{2}\right)$. This implies that the temporal correlator at zero spatial momentum $D(t, \mathbf{p}=0)$ can be written as

$$
C(t) \equiv D(t, 0)=\int_{0}^{\infty} d \omega \rho\left(\omega^{2}\right) e^{-\omega t}
$$

Clearly, a positive density $\rho\left(\omega^{2}\right)$ implies that $C(t)>0$. Notice that having $C(t)$ positive for all $t$ does not ensure the positivity of $\rho\left(\omega^{2}\right)$. On the other hand, finding $C(t)<0$ for some $t$ implies that $\rho\left(\omega^{2}\right)$ cannot be positive, suggesting confinement for the corresponding particle.

On the lattice, the real-space propagator can be evaluated using

$$
C(t)=\frac{1}{N} \sum_{k_{0}=0}^{N-1} e^{-2 \pi i k_{0} t / N} D\left(k_{0}, 0\right),
$$

where $N$ is the number of points per lattice side and $D(k)$ is the propagator in momentum space. If the lattice action satisfies reflection positivity [57], then we can write the spectral representation

$$
C(t)=\sum_{n} r_{n} e^{-E_{n} t},
$$

where $r_{n}$ are positive-definite constants. Clearly, this implies that $C(t)$ is non-negative for all values of $t$.

Numerical indications of a negative real-space lattice Landau gluon propagator have been presented in the $3 d S U(2)$ case [58], in the magnetic sector of the $4 d S U(2)$ case at finite temperature [59] and, recently, in the $4 d S U(3)$ case for one "exceptional" configuration [33].

Using data from the largest lattice sides to date, we verify explicitly (in the $3 d$ case) [32] the violation of reflection

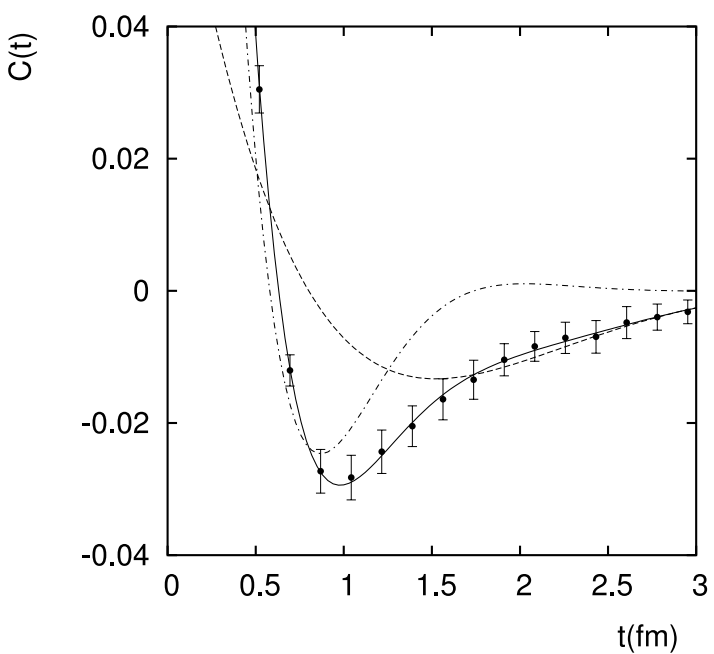

FIG. 5: Fit of $C(t)$ as a function of $t$ (in $f m$ ) using a sum of two Gribov-like propagators for lattice volume $V=140^{3}$ and coupling $\beta=4.2$. We also display the two Gribov-like propagators separately.

positivity for the $S U(2)$ lattice Landau gluon propagator (see Fig. 4). In particular, the propagator becomes negative at $t \approx 0.7 \mathrm{fm}$ and the minimum is reached at $t_{\min } \approx 1 \mathrm{fm}$ (see Fig. 5). Note that the Gribov-like propagator

$$
C(t)=\frac{e^{-M t / \sqrt{2}}}{2 M} \cos \left(\frac{M t}{\sqrt{2}}+\frac{\pi}{4}\right)
$$

has its minimum at $t_{\min }=\pi /(M \sqrt{2})$. Thus, the above result for $t_{\min }$ would imply $M \approx \pi / \sqrt{2} \mathrm{fm}^{-1} \approx 2.22 \mathrm{fm}^{-1}=$ $438 \mathrm{MeV}=0.995 \sqrt{\sigma}$, where $\sigma$ is the string tension. Let us also recall that the momentum-space Gribov-like propagator has its maximum at $p_{\max }=M$ (see Section III A above). Moreover, we find that finite-size effects seem to become important only at $t \gtrsim 3 \mathrm{fm}$. This means that our data for $t \in[0,3] \mathrm{fm}$ are essentially infinite-volume continuum results. In the scaling region, the data are well described by a sum of Gribov-like formulas (see Fig. 5), with a light-mass scale $m \approx 0.74 \sqrt{\sigma}=325 \mathrm{MeV}$.

It has been suggested $[60,61]$ that the violation of spectral positivity in lattice Landau gauge be related to the quenched auxiliary fields used for gauge fixing. We note that the fitting form proposed for $C(t)$ in [61] describes reasonably well our data up to $t=3 \mathrm{fm}$ - yielding a light-mass scale of about $1.14 \sqrt{\sigma}=500 \mathrm{MeV}$ - but cannot account for the oscillatory behavior observed at very large separations.

\section{GHOST-GLUON VERTEX}

In the framework of quantum field theory, Faddeev-Popov ghosts are introduced in order to quantize non-Abelian gauge theories. Although the ghosts are a mathematical artifact and are absent from the physical spectrum, one can use the ghostgluon vertex and the ghost propagator to calculate physical 
observables, such as the QCD running coupling $\alpha_{s}(p)$, using the relation

$$
\alpha_{s}(p)=\alpha_{0} \frac{Z_{3}(p) \widetilde{Z}_{3}^{2}(p)}{\widetilde{Z}_{1}^{2}(p)} .
$$

Here $\alpha_{0}=g_{0}^{2} / 4 \pi$ is the bare coupling constant and $Z_{3}(p)$, $\widetilde{Z}_{3}(p)$ and $\widetilde{Z}_{1}(p)$ are, respectively, the gluon, ghost and ghostgluon vertex renormalization functions. The above formula gets simplified if one considers the Landau gauge. Indeed, in this case the vertex renormalization function $\widetilde{Z}_{1}(p)$ is finite and constant, i.e. independent of the renormalization scale $p$, at least to all orders of perturbation theory $[47,62]$. Of course, it is important to verify in a non-perturbative way that this result really holds. If it does, one can consider in the Landau gauge a definition of the running coupling constant that requires only the calculation of the gluon and ghost propagators [63].

In Refs. [48] we have studied the reduced ghost-gluon vertex function $\Gamma_{\mu}^{a b c}(0, p)$ and the renormalization function $\widetilde{Z}_{1}(p)$ in minimal Landau gauge at the asymmetric point $(0 ; p,-p)$ in the $S U(2)$ case. We find that the vertex function has the same momentum dependence of the (lattice) tree-level vertex - i.e. $\sim \hat{p} \cos (\pi \tilde{p} a / L) \sim \sin (2 \pi \tilde{p} a / L)-$ and that $\widetilde{Z}_{1}(p)$ is approximately constant and equal to 1 , at least for momenta $p \gtrsim 1 \mathrm{GeV}$ (see Fig. 6). This is a direct non-perturbative verification of the perturbative result reported above. In particular, using the result obtained at the largest value of $\beta$ considered (i.e. $\beta=2.4$ ) we obtained $\widetilde{Z}_{1}^{-1}(p)=1.02_{-7}^{+6}$, where errors include Gribov-copy effects and discretization errors related to the breaking of rotational invariance.

Recently, this study has been extended (in the $3 \mathrm{~d}$ case) [65] to other kinematical configurations, including in particular the symmetric point $p^{2}=q^{2}=k^{2}$. We find that the vertex is essentially constant and of order one, for all momentum configurations (see Fig. 7), confirming the results obtained in the $4 \mathrm{~d}$ case $[45,48]$. This result is also in agreement with predictions from functional methods in the $3 \mathrm{~d}$ case [66]. In the same reference we also present the first numerical study of the Landau-gauge three-gluon vertex in the $3 \mathrm{~d}$ case and results for the spectrum of the Faddeev-Popov operator, which plays an important role in the Gribov-Zwanziger scenario [18, 28, 67]. In particular we have shown that (see Fig. 7 in Ref. [65]) the smallest non-zero eigenvalue of the Faddeev-Popov matrix goes to zero when the infinite-volume limit is approached. As a consequence, in the continuum limit, the average lattice Landau configuration should belong to the first Gribov horizon, supporting the Gribov-Zwanziger mechanism of confinement (see also [42]).

\section{GHOST CONDENSATES}

The QCD vacuum is known to be highly non-trivial at low energies [68]. This non-trivial structure manifests itself through the appearance of vacuum condensates, i.e. vacuum expectation values of certain local operators. In perturbation theory these condensates vanish, but in the SVZ-sum-

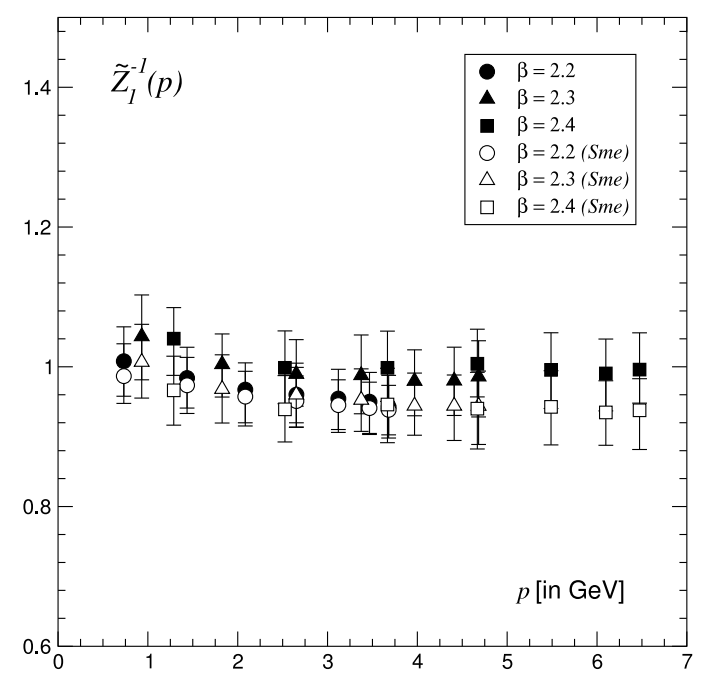

FIG. 6: Results for $\widetilde{Z}_{1}^{-1}(p)$ for the lattice volume $V=16^{4}$ as a function of $p=\hat{p} / a$ in $\mathrm{GeV}$, considering symmetric momenta, i.e. with 4 equal components. We show data obtained using two different gauge-fixing methods (with and without the so-called smearing method [64]). Error bars were evaluated using the bootstrap method with 250 samples.

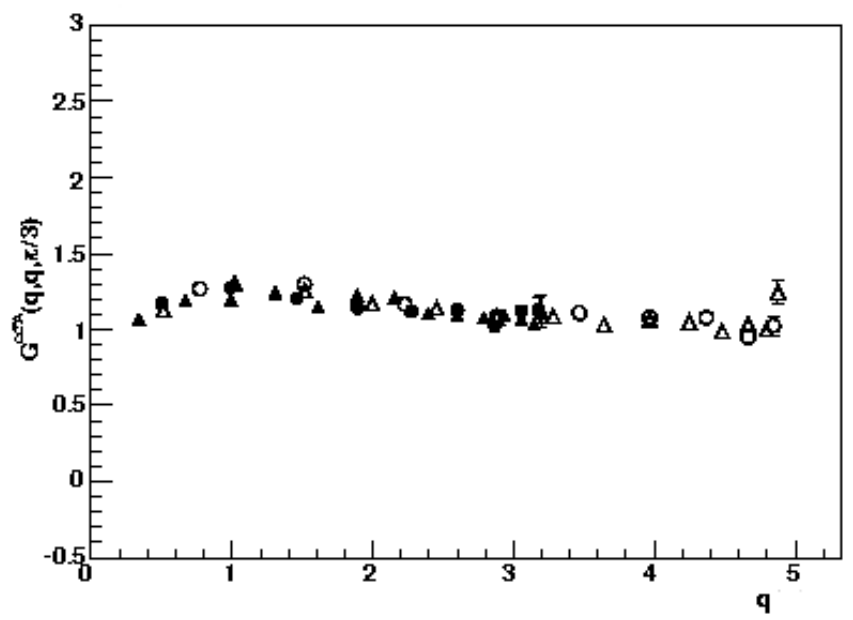

FIG. 7: The scalar quantity $G^{c \bar{c} A}(q, k, \phi)$, defined in Eq. (27) of Ref. [65], as a function of the magnitude of the incoming anti-ghost momentum $q$. Full symbols correspond to $\beta=4.2$ and open symbols to $\beta=6.0$; circles are used for $V=20^{3}$ and triangles for $V=30^{3}$. We plot data for the case with the three momenta equal.

rule approach [69] they are included as a parametrization of non-perturbative effects in the evaluation of phenomenological quantities. The two main such operators are $\alpha_{s} F_{\mu v} F^{\mu v}$ and $m_{q} \bar{\psi}_{q} \psi_{q}$; their vacuum expectation values are the so-called gluon and quark condensates. Both of these operators have mass dimension four.

In recent years, (gauge-dependent) condensates of mass di- 
mension two have also received considerable attention [29, 70-82]. In particular, the gauge condensate $\left\langle A_{\mu}^{b} A_{\mu}^{b}\right\rangle$ has been largely studied, since it should be sensitive to topological structures such as monopoles [70] and it could play an important role in the quark-confinement scenario through monopole condensation [83]. Moreover, the existence of a gauge condensate would imply a dynamical mass generation for the gluon and ghost fields [29, 71]. Possible effects of the gauge condensate $\left\langle A_{\mu}^{b} A_{\mu}^{b}\right\rangle$ on propagators and vertices (in Landau gauge) have been studied through lattice simulations in Refs. $[44,84]$, yielding $\left\langle A_{\mu}^{b} A_{\mu}^{b}\right\rangle \approx 3 \mathrm{GeV}^{2}$.

Other vacuum condensates of mass dimension two considered by several groups are the ghost condensates. These condensates were first introduced in $S U(2)$ gauge theory in maximally Abelian gauge (MAG) [72-74, 85]. More recently, the same condensates have been studied in other gauges [75, 76], such as the Curci-Ferrari and the Landau gauges. In all cases it was found that the ghost condensates are related to the breakdown of a global $S L(2, R)$ symmetry [26, 76]. In MAG the diagonal and off-diagonal components of the ghost propagators are modified $[72,77]$ by ghost condensation. Similar results have been obtained in other gauges [75, 78]. In particular, in Landau gauge it was shown [78] that the off-diagonal (antisymmetric) components of the ghost propagator $G^{c d}(p)$ are proportional to the ghost condensate $v$.

Finally, a mixed gluon-ghost condensate of mass dimension two has also been studied by several authors [73, 75, 79-81], using various gauges. This mixed condensate is of particular interest when considering interpolating gauges [80]. Indeed, it allows to generalize and relate results obtained in different

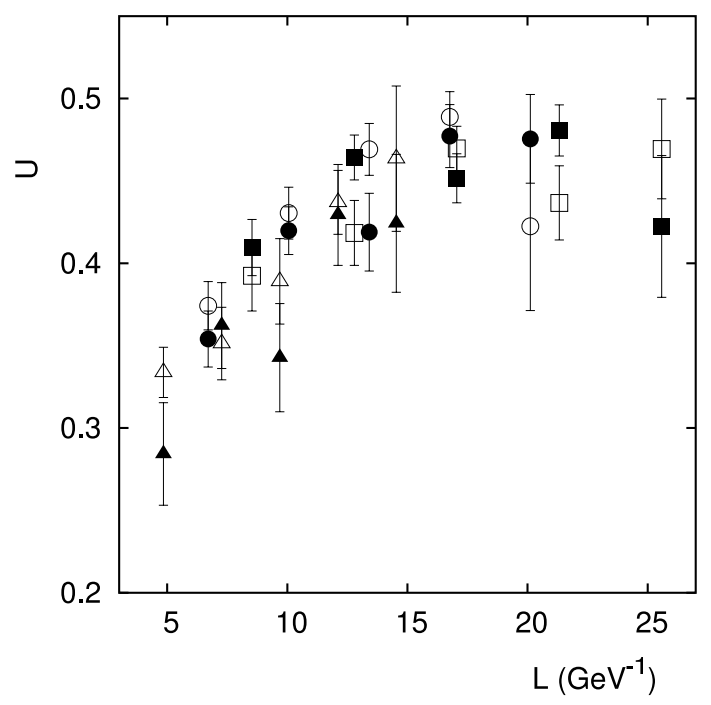

FIG. 8: Results for the Binder cumulant $U$ for the quantity $\phi^{b}(p)$ as a function of the lattice side $L$ (in $\mathrm{GeV}^{-1}$ ) for various lattice volumes $V$ and momentum with $\widetilde{p}=N / 4$. We show the data corresponding to asymmetric momenta [for $\beta=2.2(\square), \beta=2.3(\bigcirc)$ and $\beta=2.4(\triangle)$ ] and to symmetric momenta (with the corresponding filled symbols for each $\beta$ ). Errors have been estimated using the bootstrap method with 10,000 samples. gauges for the gauge condensate $\left\langle A_{\mu}^{b} A_{\mu}^{b}\right\rangle$ and the ghost condensates. Moreover, in MAG this mixed condensate would induce a dynamic mass for the off-diagonal gluons [81], giving support to the Abelian-dominance scenario [86]. Thus, the various gauge and ghost condensates could all play an important role in the dual superconducting scenario of quark confinement [87], being related to monopole condensation and to Abelian dominance.

In Ref. [88] we carried out a thorough investigation of ghost condensation in the so-called Overhauser channel for pure $S U$ (2) Yang-Mills theory in minimal Landau gauge. In particular, we evaluate numerically the off-diagonal components of the ghost propagator $G^{c d}(p)$ as a function of the momentum $p$. We find that $\left\langle\phi^{b}(p)\right\rangle=\varepsilon^{b c d}\left\langle G^{c d}(p)\right\rangle / 2$ is zero within error bars, but with large fluctuations. At the same time, we see clear signs of spontaneous breaking of a global symmetry, using the quantity $\phi^{b}(p)$ as an order parameter. As in the case of continuous-spin models in the ordered phase (see for example [89]), spontaneous symmetry breaking is supported by two (related) observations: 1) by comparing the statistical fluctuations for the quantities $\phi^{b}(p)$ and $\left|\phi^{b}(p)\right|$; 2) from the nonGaussian shape of the statistical distribution of $\phi^{b}(p)$, which can be observed by considering a histogram of the data or by evaluating the so-called Binder cumulant (see Fig. 8). Since, in Landau gauge, the vacuum expectation value of the quantity $\phi^{b}(p)$ should be proportional (in the Overhauser channel) to the ghost condensate $v$ [78], it seems reasonable to conclude that the broken symmetry is the $\operatorname{SL}(2, R)$ symmetry, which is related to ghost condensation $[26,76]$. This interpretation has been recently criticized in Ref. [90]. There, the nonzero value obtained for the Binder cumulant has been explained by considering multi-dimensional Gaussian distributions and a modified definition for the Binder cumulant. We note however that the order parameter considered in our study, i.e. the magnitude of $\phi^{b}(p)$, is a scalar quantity. Therefore, in analogy with studies of $O(N)$-vector models (see for example [91]), the standard definition of the Binder cumulant should apply to our study as well.

Let us note that, from our data, the Binder cumulant $U$ seems to be approximately null at small lattice volume and to converge to a value $U \approx 0.45$ for physical lattice side $L \gtrsim 15$ $\mathrm{Gev}^{-1} \approx 3 \mathrm{fm}$, corresponding to a mass scale of less than 100 $\mathrm{MeV}$.

In Ref. [88] we have also shown that the sign of $\phi^{b}(p)$ is related to the sign of the Fourier-transformed gluon field components $\widetilde{A}(q)$ and that $\phi^{b}(p)$ has discretization effects similar to those obtained for the ghost-gluon vertex [48]. Then, using the rescaled quantity $\left|L^{2} \phi^{b}(p) / \cos (\pi \tilde{p} a / L)\right|$, we find (at small momenta) a behavior $p^{-z}$ with $z \approx 4$, in agreement with analytic predictions [78]. On the other hand, from our fits we find that the ghost condensate $v$ is consistent with zero within error bars, i.e. the quantity $\left|L^{2} \phi^{b}(p) / \cos (\pi \tilde{p} a / L)\right|$ does not approach a finite limit at small momenta, at least for $p \geq 0.245 \mathrm{GeV}$. Using the Ansatz

$$
G^{c d}(p)=\frac{p^{2} \delta^{c d}+v \varepsilon^{c d}}{p^{4}+v^{2}},
$$

we obtain for the ghost condensate the upper bound $v \ll 0.058$ 


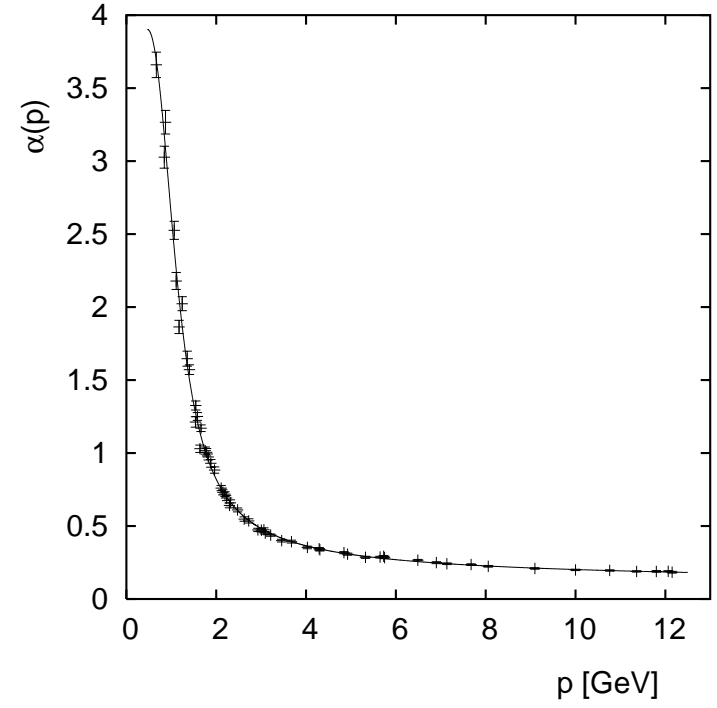

FIG. 9: Fit for the running coupling $\alpha_{s}(p)$ using eq. (10) with $C=$ $0.072(8), a=1.9(3), \Lambda=1.31(1)$ and $m=1.0(6)$.

$\mathrm{GeV}^{2}$. More precisely, our data rule out values of $v$ greater than $0.058 \mathrm{GeV}^{2} \approx(240 \mathrm{MeV})^{2}$ but would still be consistent with a ghost condensate $v \lesssim 0.025 \mathrm{GeV}^{2} \approx(160 \mathrm{MeV})^{2}$. Let us note that, in analytic studies $[73,78,82,92]$ one finds that the ghost condensate induces a tachyonic gluon mass proportional to $\sqrt{v}$, which modifies the dynamic gluon mass related to the gauge condensate $\left\langle A_{\mu}^{b} A_{\mu}^{b}\right\rangle$. Thus, one should expect a relatively small ghost condensate $v$ in order to obtain a global (non-tachyonic) gluon mass. Let us recall that a dynamic gluon mass of the order of a few hundred $\mathrm{MeV}$ has been considered in several phenomenological studies [93]. A similar mass scale was also obtained in numerical studies of the gluon propagator in Landau gauge [32, 39, 51].

\section{RUNNING COUPLING}

Of great importance for phenomenological purposes is the running coupling strength $\alpha_{s}(p)$ [63] defined in Eq. (6) above. In particular, this quantity enters directly the quark DysonSchwinger equation (DSE) and can be interpreted as an effective interaction strength between quarks [94]. Let us note that, working in Landau gauge and in the momentum-subtraction scheme, the running coupling (6) can be written as [63]

$$
\alpha_{s}(p)=\alpha_{0} F(p) J^{2}(p),
$$

where $F(p)$ and $J(p)$ are, respectively, the gluon and the ghost form factors and we used the result $\widetilde{Z}_{1}(p)=1$. As explained in Sec. II above, studies using DSE's have found that, if the IR sum rule $2 a_{G}-a_{D}=0$ is satisfied, then this running coupling develops a fixed point in the IR limit (see for example [35])

$$
\lim _{p \rightarrow 0} \alpha_{s}(p)=\alpha_{c}=\text { constant }
$$

with $\alpha_{c} \approx 8.92 / N_{c}$ in the $\operatorname{SU}\left(N_{c}\right)$ case for $\kappa \approx 0.596$.

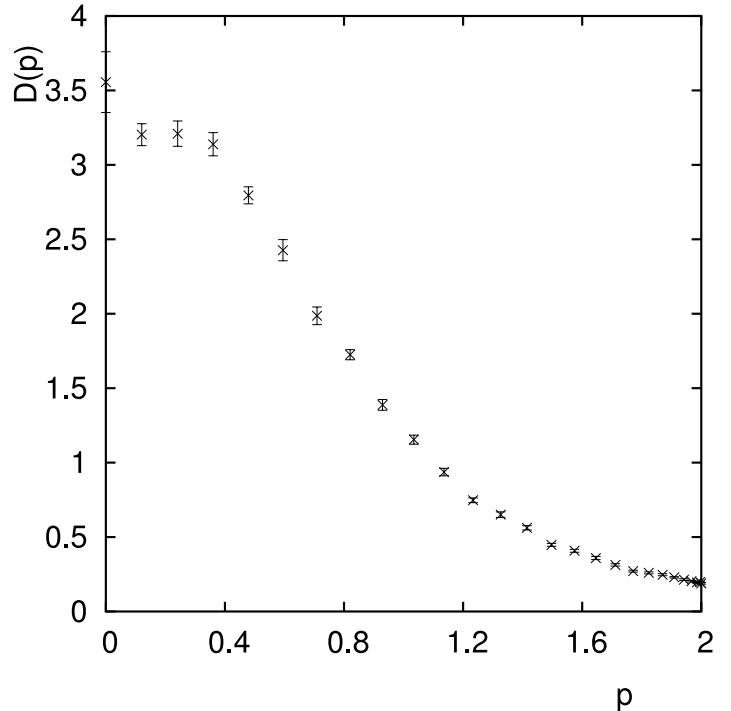

FIG. 10: Plot of the gluon propagator $D(p)$ as a function of $p$ for lattice volume $V=52^{4}$ at $\beta=2.2$. All quantities are in lattice units (here $a \approx 1 \mathrm{GeV}^{-1}$ ). Errors represent one standard deviation. The larger value at $p=0$ is probably due to an insufficient number of gauge-fixing iterations.

This quantity has been studied numerically by several groups (see for example [33, 44, 95]). In Ref. [96] we have evaluated this running coupling constant and tried a fit to the data using the fitting function

$$
\alpha_{s}(p)=C p^{4} /\left[\left(p^{4}+m\right) s(a)\right]
$$

where $s(a)=\left(11 / 24 \pi^{2}\right) \log \left[1+\left(p^{2} / \Lambda^{2}\right)^{a}\right]$. Note that if $a=$ 2 one finds a fixed point in the IR limit equal to $\alpha_{c}=$ $24 \pi^{2} C \Lambda^{4} / 11 \mathrm{~m}$. Also, this fitting functions satisfies the leading ultraviolet behavior $\sim 1 / \log \left[\left(p^{2} / \Lambda^{2}\right)\right]$ for the running coupling. Results are reported in Fig. 9; we find $a=1.9(3)$ and $\alpha_{c}=24 \pi^{2} C \Lambda^{2} / 11 m \approx 4.6$, in agreement with the result reported above. Similar results have also been obtained in [38]. We stress that in Refs. [38, 96] we have compared results obtained using two slightly different lattice formulations, yielding consistent results in all cases considered.

As said in Section II above, recent lattice studies [44, 45] seem to indicate a null IR limit for $\alpha_{s}(p)$, instead of a finite nonzero value $\alpha_{c}$. We should stress, however, that in these studies special care must be taken in order to eliminate finite-size effects, especially when the IR region is considered. Indeed, we know that, if the the gluon propagator is not suppressed at small momenta, the true infrared regime is surely not reached yet. This is a difficult numerical task since we have checked that in the $4 \mathrm{~d} S U(2)$ case a lattice volume $V=52^{4}$ at $\beta=2.2$, corresponding to a physical lattice side of about $10 \mathrm{fm}$, is still not sufficient to show a decreasing propagator in the limit of small momenta (see Fig. 10). We note that in Fig. 1 of [44] and in Fig. 3 of [45] the running coupling starts to decrease for a momentum $p \approx 0.5 \mathrm{GeV}$. Thus, 
from our discussion in Section III A above, considering the results shown in Fig. 10 and also the results reported in [50, 52], we can say that the data considered in $[44,45]$ for momenta $p \lesssim 500 \mathrm{MeV}$ are most likely affected by strong finite-size effects.

\section{CONCLUSIONS}

The Gribov-Zwanziger and the Kugo-Ojima confinement scenarios in Landau gauge are well supported, at the qualitative level, by several studies based on different analytical and numerical approaches. On the other hand, as we have shown here, a comprehensive analysis of these confinement scenarios at the quantitative level using lattice numerical simulations could represent a very challenging task.

\section{ACKNOWLEDGMENTS}

The authors thank the organizers for the invitation. Research supported by Fundação de Amparo à Pesquisa do Estado de São Paulo (FAPESP) (under grant \# 00/05047-5). Partial support from Conselho Nacional de Desenvolvimento Científico e Tecnológico (CNPq) is also acknowledged.
[1] An Elementary Primer for Gauge Theory, K. Moriyasu (World Scientific, Singapore, 1983).

[2] K.G. Wilson, Phys. Rev. D 10, 2445 (1974); Lattice gauge theories. An introduction, H.J. Rothe (World Scientific, Singapore, 1997).

[3] A guide to Monte Carlo simulations in Statistical Physics, D.P Landau and K. Binder (Cambridge University Press, Cambridge UK, 2000).

[4] Parallel computer architecture: a hardware/software approach, D. E. Culler, J.P. Singh, and A. Gupta (Morgan Kaufmann, San Francisco CA, 1999).

[5] K. Kanaya, Fortsch. Phys. 50, 531 (2002).

[6] R.D. Mawhinney, Parallel Comput. 25, 1281 (1999); P.A. Boyle et al., J. Phys. Conf. Ser. 16, 129 (2005).

[7] A. Bartoloni et al., Nucl. Phys. Proc. Suppl. A 60, 237 (1998); R. Ammendola et al., Nucl. Phys. Proc. Suppl. B 119, 1038 (2003).

[8] M. Lüscher, Nucl. Phys. Proc. Suppl. B 106, 21 (2002).

[9] Proceedings of the XXIIIrd International Symposium on Lattice Field Theory, PoS LAT2005 and previous years.

[10] M. Creutz, AIP Conf. Proc. 690, 52 (2003).

[11] A. Cucchieri, in the Proceedings of Hadron Physics 2002, edited by C.A.Z. Vasconcellos et al. (World Scientific, Cingapura, 2003) 137.

[12] Quantum chromodynamics, I. Hinchliffe, in Review of particle physics, Particle Data Group (K. Hagiwara et al.), Phys. Rev. D 66 (2002) 010001.

[13] S. Aoki et al., Phys. Rev. Lett. 84, 238 (2000).

[14] F. Karsch, Lect. Notes Phys. 583, 209 (2002).

[15] F. Wilczek, Nucl. Phys. Proc. Suppl. B 119, 3 (2003).

[16] C.T.H. Davies et al., Phys. Rev. Lett. 92, 022001 (2004); P. Lepage and C. Davies, Int. J. Mod. Phys. A 19, 877 (2004).

[17] http://lattice.ifsc.usp.br/.

[18] V.N. Gribov. Nucl. Phys. B 139, 1 (1978).

[19] D. Zwanziger. Nucl. Phys. B 412, 657 (1994).

[20] A. Cucchieri and T. Mendes, Nucl. Phys. B 471, 263 (1996); Nucl. Phys. Proc. Suppl. B 53, 811 (1997); Phys. Rev. D 57, 3822(R) (1998); Comput. Phys. Commun. 154, 1 (2003).

[21] A. Cucchieri, Nucl. Phys. B 508, 353 (1997).

[22] T. Mendes and A. Cucchieri, AIP Conf. Proc. 739, 599 (2004); T. Mendes, Nucl. Phys. A 702, P29 (2002); in the Proceedings of Hadron Physics 2002, edited by C.A.Z. Vasconcellos et al. (World Scientific, Cingapura, 2003) 297; AIP Conf. Proc. 756, 413 (2005).

[23] A. Cucchieri and T. Mendes, J. Phys. A 38, 4561 (2005); Nu- merical simulation of $N$-vector spin models in a magnetic field, to appear in Braz. J. Phys.

[24] W.G. Wanzeller, A. Cucchieri, G. Krein, and T. Mendes, Braz. J. Phys. 34, 247 (2004).

[25] Dynamic behavior of cluster observables for the $2 d$ Ising model, W.G. Wanzeller, T. Mendes, and G. Krein, to appear in Braz. J. Phys; W.G. Wanzeller, T. Mendes, and G. Krein, cond-mat/0605438.

[26] R. Alkofer and L. von Smekal, Phys. Rept. 353, 281 (2001).

[27] A. Holl, C.D. Roberts, and S.V. Wright, nucl-th / 0601071.

[28] D. Zwanziger, Nucl. Phys. B 412, 657 (1994).

[29] R.F. Sobreiro, S.P. Sorella, D. Dudal, and H. Verschelde, Phys. Lett. B 590, 265 (2004).

[30] T. Kugo and I. Ojima, Prog. Theor. Phys. Suppl. 66, 1 (1979); Erratum - Prog. Theor. Phys. 71, 1121 (1984); T. Kugo, hep-th/9511033.

[31] D. Zwanziger, Phys. Lett. B 257, 168 (1991); Nucl. Phys. B 364, 127 (1991); Nucl. Phys. B 378, 525 (1992).

[32] A. Cucchieri, T. Mendes, and A.R. Taurines, Phys. Rev. D 71, 051902(R) (2005).

[33] S. Furui and H. Nakajima, Phys. Rev. D 70, 094504 (2004).

[34] D. Zwanziger, Phys. Rev. D 65, 094039 (2002).

[35] C. Lerche and L. von Smekal, Phys. Rev. D 65, 125006 (2002).

[36] R. Alkofer, W. Detmold, C.S. Fischer, and P. Maris, Phys. Rev. D 70, 014014 (2004).

[37] H. Suman and K. Schilling, Phys. Lett. B 373, 314 (1996); S. Furui and H. Nakajima, Phys. Rev. D 69, 074505 (2004).

[38] J.C.R. Bloch, A. Cucchieri, K. Langfeld, and T. Mendes, Nucl. Phys. B 687, 76 (2004).

[39] A. Cucchieri, T. Mendes, and A.R. Taurines, Phys. Rev. D 67, 091502(R) (2003).

[40] O. Oliveira and P.J. Silva, AIP Conf. Proc. 756, 290 (2005); PoS LAT2005, 286 (2005); PoS LAT2005, 287 (2005); hep-lat 10511043.

[41] A. Cucchieri and D. Zwanziger, Phys. Rev. D 65, 014001 (2002); Phys. Lett. B 524, 123 (2002).

[42] A. Cucchieri, Nucl. Phys. B 521, 365 (1998).

[43] H. Nakajima, S. Furui, and A. Yamaguchi, hep-lat/0007001; S. Furui and H. Nakajima, hep-lat/0403021; Phys. Rev. D 73, 074503 (2006).

[44] P. Boucaud et al., hep-ph/0507104.

[45] E.M. Ilgenfritz, M. Muller-Preussker, A. Sternbeck, and A. Schiller, hep-lat/0601027.

[46] C.S. Fischer, B. Gruter, and R. Alkofer, hep-ph/0506053.

[47] J.C. Taylor, Nucl. Phys. B 33, 436 (1971). 
[48] A. Cucchieri, T. Mendes, and A. Mihara, JHEP 0412, 012 (2004); AIP Conf. Proc. 739, 602 (2005).

[49] A. Cucchieri, T. Mendes, G. Travieso, and A.R. Taurines, hep-lat/0308005, in the Proceedings of the 15th Symposium on Computer Architecture and High Performance Computing, edited by L.M. Sato et al. (IEEE Computer Society Press, Los Alamitos CA, 2003) 123.

[50] D.B. Leinweber et al., Phys. Rev. D 60, 094507 (1999) [Erratum-ibid. D 61, 079901 (2000)].

[51] A. Cucchieri, Phys. Rev. D 60, 034508 (1999).

[52] F.D. Bonnet et al., Phys. Rev. D 64, 034501 (2001).

[53] A. Cucchieri, A. Maas, and T. Mendes, in preparation.

[54] M.B. Parappilly et al., hep-lat/0601010.

[55] A. Cucchieri and T. Mendes, Phys. Rev. D 73, 071502(R) (2006).

[56] H. Aiso et al., Nucl. Phys. Proc. Suppl. B 53, 570 (1997).

[57] Quantum Fields on a Lattice, I. Montvay and G. Münster (Cambridge University Press, Cambridge UK, 1994).

[58] A. Cucchieri, F. Karsch, and P. Petreczky, Phys. Lett. B 497, 80 (2001); Nucl. Phys. Proc. Suppl. B 94, 385 (2001).

[59] A. Cucchieri, F. Karsch, and P. Petreczky, Phys. Rev. D 64, 036001 (2001).

[60] C.A. Aubin and M.C. Ogilvie, Phys. Lett. B 570, 59 (2003).

[61] C. Aubin and M.C. Ogilvie, Phys. Rev. D 70, 074514 (2004).

[62] O. Piguet and S.P. Sorella, Lect. Notes Phys. M 28, 1 (1995).

[63] L. von Smekal, R. Alkofer, and A. Hauck, Phys. Rev. Lett. 79, 3591 (1997); Annals Phys. 267, 1 (1998) [Erratum-ibid. 269, 182 (1998)].

[64] J.E. Hetrick and P. de Forcrand, Nucl. Phys. Proc. Suppl. B 63, 838 (1998).

[65] A. Cucchieri, A. Maas, and T. Mendes, hep-lat/0605011.

[66] W. Schleifenbaum, A. Maas, J. Wambach, and R. Alkofer, Phys. Rev. D 72, 014017 (2005); W. Schleifenbaum, M. Leder, and H. Reinhardt, hep-th/0605115.

[67] A. Sternbeck, E. M. Ilgenfritz, and M. Müller-Preussker, Phys. Rev. D 73, 014502 (2006).

[68] The QCD vacuum, hadrons and the superdense matter, E.V. Shuryak (World Scientific, Singapore, 1988).

[69] M.A. Shifman, A.I. Vainshtein, and V.I. Zakharov, Nucl. Phys. B 147, 385 (1979); Nucl. Phys. B 147, 448 (1979).

[70] F.V. Gubarev, L. Stodolsky, and V.I. Zakharov, Phys. Rev. Lett. 86, 2220 (2001).

[71] K.I. Kondo, Phys. Lett. B 560, 44 (2003); D. Dudal et al., JHEP 0401, 044 (2004); D. Dudal, R.F. Sobreiro, S.P. Sorella, and H. Verschelde, Phys. Rev. D 72, 014016 (2005).

[72] K.I. Kondo and T. Shinohara, Phys. Lett. B 491, 263 (2000).

[73] D. Dudal and H. Verschelde, J. Phys. A 36, 8507 (2003).

[74] K.I. Kondo, Phys. Lett. B 572, 210 (2003).

[75] K.I. Kondo, T. Murakami, T. Shinohara, and T. Imai, Phys. Rev. D 65, 085034 (2002).

[76] D. Dudal et al., JHEP 0212, 008 (2002).
[77] V.E.R. Lemes, M.S. Sarandy, and S.P. Sorella, J. Phys. A 36, 7211 (2003).

[78] D. Dudal et al., JHEP 0306, 003 (2003).

[79] K.I. Kondo, Phys. Lett. B 514, 335 (2001); D. Dudal et al., Annals Phys. 308, 62 (2003).

[80] D. Dudal et al., Phys. Rev. D 70, 114038 (2004).

[81] D. Dudal et al., hep-th/0501227.

[82] M.A.L. Capri et al., Phys. Rev. D 73, 014001 (2006).

[83] G. 't Hooft, Nucl. Phys. B 190, 455 (1981); T. Suzuki, Nucl. Phys. Proc. Suppl. B 30, 176 (1993).

[84] P. Boucaud et al., Phys. Rev. D 63, 114003 (2001); Phys. Rev. D 66, 034504 (2002); Phys. Rev. D 67, 074027 (2003); hep-lat/0504017; E. Ruiz Arriola, P.O. Bowman, and W. Broniowski, Phys. Rev. D 70, 097505 (2004); S. Furui and H. Nakajima, hep-lat/0503029.

[85] M. Schaden, hep-th/9909011; hep-th/0108034 , in the Proceedings of 4th International Conference on Quark confinement and the hadron spectrum, edited by W. Lucha and K.M. Maung (World Scientific, Singapore, 2001) 258.

[86] Z.F. Ezawa and A. Iwazaki, Phys. Rev. D 25, 2681 (1982); T. Suzuki, Prog. Theor. Phys. 81, 752 (1989).

[87] Y. Nambu, Phys. Rev. D 10, 4262 (1974); S. Mandelstam, Phys. Rept. 23, 245 (1976).

[88] A. Cucchieri, T. Mendes, and A. Mihara, Phys. Rev. D 72, 094505 (2005).

[89] Monte Carlo Simulation in Statistical Physics An Introduction, K. Binder and D.W. Heermann (Springer-Verlag, Berlin, 2002); Computer Studies of Phase Transitions and Critical Phenomena, O.G. Mouritsen (Springer-Verlag, Berlin, 1984).

[90] S. Furui and H. Nakajima, hep-lat/0602027.

[91] K. Kanaya and S. Kaya, Phys. Rev. D 51, 2404 (1995); J. Engels and T. Mendes, Nucl. Phys. B 572, 289 (2000).

[92] H. Sawayanagi, Phys. Rev. D 67, 045002 (2003).

[93] G. Parisi and R. Petronzio, Phys. Lett. B 94, 51 (1980); F. Halzen, G.I. Krein, and A.A. Natale, Phys. Rev. D 47, 295 (1993); M.B. Gay Ducati, F. Halzen, and A.A. Natale, Phys. Rev. D 48, 2324 (1993); J.H. Field, Phys. Rev. D 66, 013013 (2002); M.B. Gay Ducati and W.K. Sauter, hep-ph/0501057.

[94] J.C.R. Bloch, Phys. Rev. D 66, 034032 (2002).

[95] A. Cucchieri, T. Mendes, and D. Zwanziger, Nucl. Phys. Proc. Suppl. B 106, 697 (2002); K. Langfeld et al., hep-th/0209173, in the Proceedings of Gargnano 2002, Quark confinement and the hadron spectrum, edited by N. Brambilla and G. M. Prosperi (World Scientific, River Edge, 2003) 297; H. Nakajima and S. Furui, Nucl. Phys. Proc. Suppl. 129, 730 (2004); A. Sternbeck, E.M. Ilgenfritz, M. MullerPreussker, and A. Schiller, PoS LAT2005, 333 (2006).

[96] J.C.R. Bloch, A. Cucchieri, K. Langfeld, and T. Mendes, Nucl. Phys. Proc. Suppl. B 119, 736 (2003). 